\title{
Diffraction on nuclei: effects of nucleon-nucleon correlations and inelastic shadowing within an improved Glauber-Gribov approach
}

\author{
Claudio Ciofi degli Atti*i \\ INFN, Sezione di Perugia, Via A. Pascoli, Perugia, Italy \\ E-mail: ciofi@pg.infn.it
}

The importance of the effects of nucleon-nucleon (NN) short-range correlations (SRC) and Gribov inelastic shadowing (IS) on various high energy scattering processes involving nuclear targets is demonstrated within an improved Glauber-Gribov approach.

Sixth International Conference on Quarks and Nuclear Physics April 16-20, 2012

Ecole Polytechnique, Palaiseau, Paris

\footnotetext{
* Speaker.

${ }^{\dagger}$ Collaboration between INFN, Sezione di Perugia and Departamento de Física, Universidad Tecnica Federico Santa María (UTFSM), Valparaíso, Chile.
} 


\section{Contents}

1. Introduction 2

2. Formal approach 2

3. Results of calculations 5

4. Acknowledgments 6

\section{Introduction}

It is well known that high energy processes involving nuclear targets, namely hadron-nucleus $(h-A)$ and nucleus-nucleus $(A A)$ scattering, provide useful information on several phenomena like, e.g., hadronization and confinement, hadron propagation in medium, mechanisms of formation of high density matter, and many other ones. Most of theoretical approaches to describe scattering at multi $\mathrm{GeV}$ energies involving nuclei, are based upon Glauber multiple scattering theory [1] within the independent particle model description of the nucleus. The latter approximation seems to be out of date, for the nucleus is a self bound saturated liquid where nuclear constituents spend part of their time in strongly correlated configurations [2], which have been recently experimentally investigated in a quantitative way [3]. Besides nucleon-nucleon (NN) short-range correlations (SRC), intermediate hadron-hadron inelastic scattering (Gribov inelastic shadowing (IS) [4]), lacking in the Glauber approach, have to be considered. The importance of the effects of both SRC and Gribov IS in several high energy scattering processes have been studied in a series of recent papers [5]-[10], finding that the two effects act frequently in the opposite directions. The main results of these papers will be concisely illustrated in the following Sections.

\section{Formal approach}

The nuclear quantity entering most Glauber-like calculations is the modulus squared of the nuclear wave function $\left|\psi_{0}\right|^{2}$, whose exact expansion is usually truncated at lowest order (singledensity approximation) viz

$$
\left|\psi_{0}\left(\mathbf{r}_{1}, \ldots, \mathbf{r}_{A}\right)\right|^{2}=\prod_{j=1}^{A} \rho_{1}\left(\mathbf{r}_{j}\right)+\sum_{i<j} \Delta\left(\mathbf{r}_{i}, \mathbf{r}_{j}\right) \prod_{k \neq i, j} \rho_{1}\left(\mathbf{r}_{k}\right)+\ldots . \simeq \prod_{j=1}^{A} \rho_{1}\left(\mathbf{r}_{j}\right) .
$$

Here the two-body contraction (two-body correlation function) $\Delta\left(\mathbf{r}_{i}, \mathbf{r}_{j}\right)=\rho_{2}\left(\mathbf{r}_{i}, \mathbf{r}_{j}\right)-\rho_{1}\left(\mathbf{r}_{i}\right) \rho_{1}\left(\mathbf{r}_{j}\right)$, contains the effect of SRC, represented by a hole in the two-body density $\rho_{2}$ at short inter-nucleon 


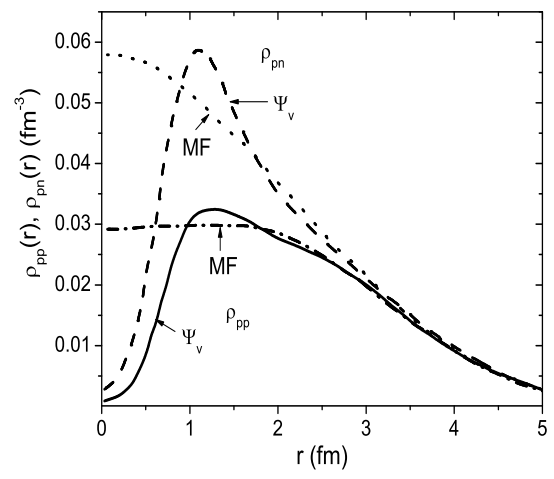

Figure 1: The proton-neutron $(p n)$ and proton-proton $(p p)$ two-body densities $\rho_{2} \equiv \rho_{N N}$ in ${ }^{16} O$ calculated [2] within a mean field model (MF) and by solving the many-body problem with a realistic NN interaction $\left(\Psi_{v}\right)$. The behavior of $\rho_{N N}$ at relative distances $r \leq 1.5 \div 2 \mathrm{fm}$ is governed by the repulsive short-range core and by the attractive intermediate-range tensor force (after Ref. [2]).

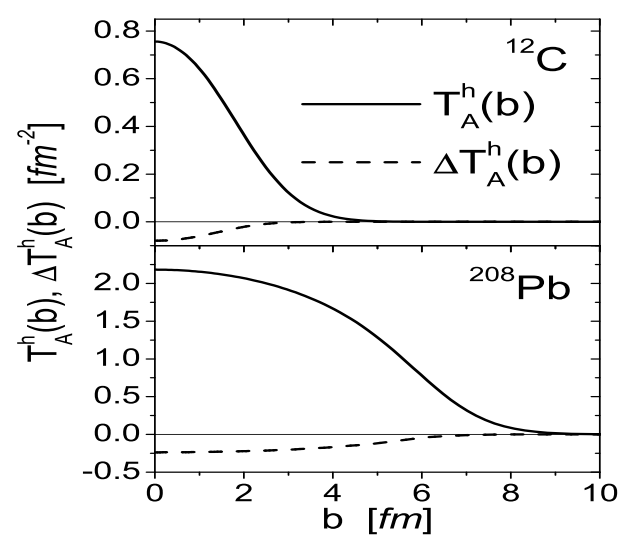

Figure 2: The thickness function $T_{A}^{h}(b)$ and the correlation contribution $\left(\Delta T_{A}^{h}(b)\right)$ in $p-{ }^{12} C$ and $p-{ }^{208} P b$ collisions at HERA-B energies. The total thickness function is given by $\widetilde{T}_{A}^{h}=T_{A}^{h}-\Delta T_{A}^{h}$ (after Ref.[6])

separations (see Fig. 1). It has been shown [5] that SRC lead to an additional contribution to the nuclear thickness function as follows $\left(\mathbf{r}_{i}=\left\{\mathbf{s}_{i}, z_{i}\right\}\right)[5]$

$$
\Delta T_{A}^{h}(b)=\frac{1}{\sigma_{\text {tot }}^{h N}} \int d^{2} \mathbf{s}_{1} d^{2} \mathbf{s}_{2} \Delta_{A}^{\perp}\left(\mathbf{s}_{1}, \mathbf{s}_{2}\right) \operatorname{Re} \Gamma^{p N}\left(\mathbf{b}-\mathbf{s}_{1}\right) \operatorname{Re} \Gamma^{p N}\left(\mathbf{b}-\mathbf{s}_{2}\right),
$$

where $\Delta_{A}^{\perp}\left(\mathbf{s}_{1}, \mathbf{s}_{2}\right)$ represents the transverse two-nucleon contraction and the total thickness function is $\widetilde{T}_{A}^{h}=T_{A}^{h}-\Delta T_{A}^{h}$. The thickness functions of ${ }^{12} C$ and ${ }^{208} \mathrm{~Pb}$ at HERA-B energies calculated with realistic two-body densities from Ref. [11] are shown in Fig. 2. SRC increase the thickness functions and, consequently, the total neutron-nucleus cross section at high energies, making the nucleus more opaque [5], unlike Gribov IS corrections which increase nuclear transparency. 


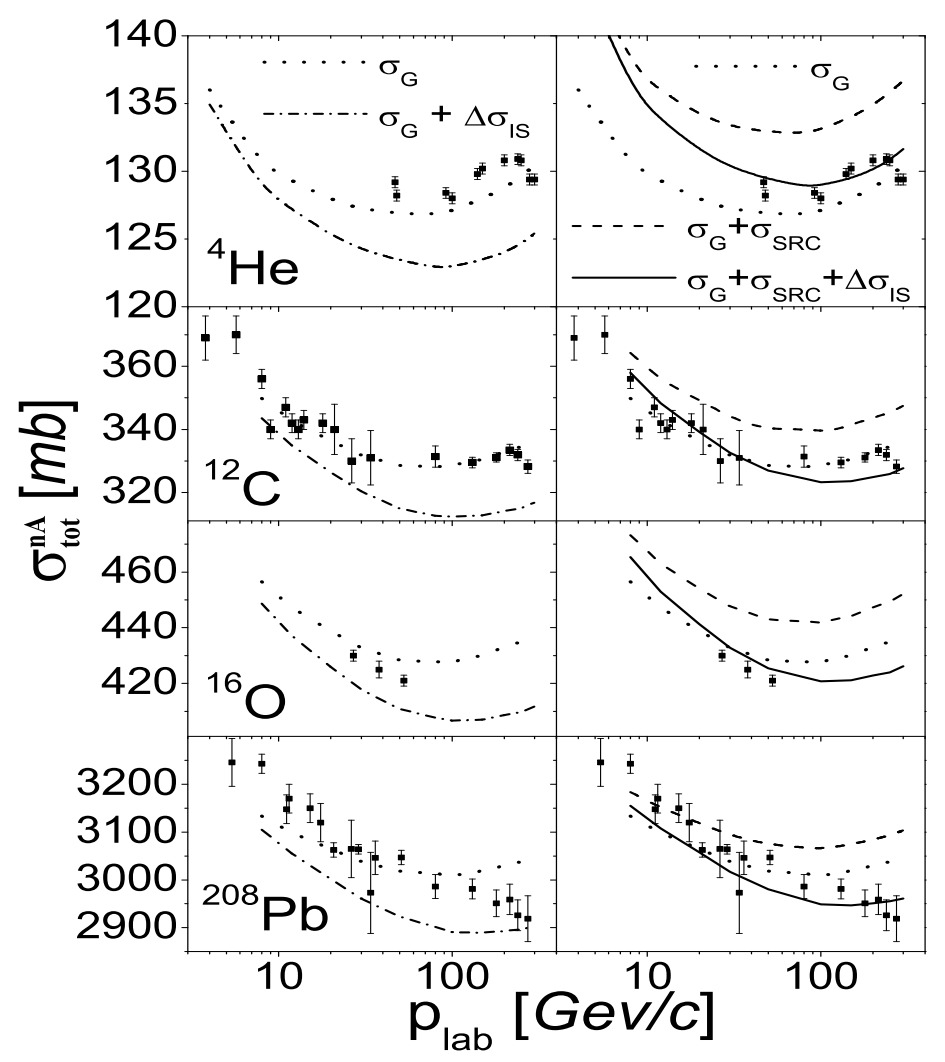

Figure 3: The total neutron-Nucleus cross section $\sigma_{\text {tot }}^{n A} v s$ plab. Left panel: Glauber single density approximation $\left(\sigma_{G}\right.$; dots $)$ and Glauber plus Gribov inelastic shadowing $\left(\sigma_{G}+\Delta \sigma_{I S} ;\right.$ dot-dash $)$. Right panel: Glauber $\left(\sigma_{G} ;\right.$ dots $)$; Glauber plus SRC $\left(\sigma_{G}+\sigma_{S R C} ;\right.$ dashes $)$; Glauber plus SRC plus Gribov inelastic shadowing $\left(\sigma_{G}\right.$ $+\sigma_{S R C}+\Delta \sigma_{I S} ;$ full $)($ after Ref. [5]).

\begin{tabular}{c|cccc}
\hline \hline${ }^{208} \mathrm{~Pb}$ & Glauber & $\begin{array}{c}\text { Glauber } \\
+ \text { SRC }\end{array}$ & $\begin{array}{c}\text { q-2q model } \\
+ \text { SRC }\end{array}$ & $\begin{array}{c}\text { 3q model } \\
+ \text { SRC }\end{array}$ \\
\hline$\sigma_{t o t}^{N A}[\mathrm{mb}]$ & 3850.63 & 3885.77 & 3833.26 & 3839.26 \\
\hline$\sigma_{e l}^{N A}[\mathrm{mb}]$ & 1664.76 & 1690.48 & 1655.70 & 1660.67 \\
\hline$\sigma_{s d}^{N A}[\mathrm{mb}]$ & - & - & 2.62 & 0.59 \\
\hline$\sigma_{s d+g}^{N A}[\mathrm{mb}]$ & - & - & 2.58 & 2.56 \\
\hline$\sigma_{q e}^{N A}[\mathrm{mb}]$ & 120.92 & 112.65 & 113.37 & 113.88 \\
\hline$\sigma_{q s d}^{N A}[\mathrm{mb}]$ & - & - & -2.08 & -2.62 \\
\hline$\sigma_{t s d}^{N A}[\mathrm{mb}]$ & - & - & 17.55 & 17.63 \\
\hline$\sigma_{d d}^{N A}[\mathrm{mb}]$ & - & - & -2.08 & -2.62 \\
\hline \hline
\end{tabular}

Table 1: Various $p-{ }^{208} \mathrm{~Pb}$ cross sections at LHC energies calculated with two models of the light-cone dipole cross section (after Ref. [6]). 


\section{Results of calculations}

An exhaustive calculation of the total, $\sigma_{t o t}^{h A}$, elastic, $\sigma_{e l}^{h A}$, quasi-elastic, $\sigma_{q e l}^{h A}$, inelastic, $\sigma_{i n}^{h A}$, and diffractive dissociation hadron-nucleus cross sections, which include both SRC and Gribov IS summed to all orders by the light-cone dipole approach [7, 8, 9], has been performed in Ref. $[5,6,10]$ demonstrating the opposite roles played by SRC and IS. It has been found that the total contribution to the thickness function due to SRC and Gribov IS reads as follows

$$
\begin{aligned}
& \Delta T_{A}^{d i p}\left(b, \mathbf{r}_{T}, \alpha\right)= \\
& =\frac{1}{\sigma_{d i p}\left(r_{T}\right)} \int d^{2} \mathbf{s}_{1} d^{2} \mathbf{s}_{2} \Delta_{A}^{\perp}\left(\mathbf{s}_{1}, \mathbf{s}_{2}\right) \operatorname{Re} \Gamma^{\bar{q} q, N}\left(\mathbf{b}-\mathbf{s}_{1}, \mathbf{r}_{T}, \alpha\right) \operatorname{Re} \Gamma^{\bar{q} q, N}\left(\mathbf{b}-\mathbf{s}_{2}, \mathbf{r}_{T}, \alpha\right) .
\end{aligned}
$$

where $\Gamma^{q \bar{q}, N}$ is the $(q \bar{q})-N$ profile, $\sigma_{d i p}\left(r_{T}\right)$ the dipole cross section, $r_{T}$ the dipole transverse separation and $\alpha$ its fractional light-cone momentum. SRC and Gribov IS affect also the number of inelastic collisions $N_{c o l l}^{h A}=A \sigma_{i n}^{h N} / \sigma_{i n}^{h A}$, which is the normalization factor used to obtain the nucleus to nucleon ratio of the cross section of a hard reaction. The results of calculations, performed with realistic one- and two-body densities and correlation functions from Ref. [11], are shown in Fig. 3, and in Tables 1 and 2. The behavior of $N_{\text {coll }}^{N A}$ is entirely governed by the non-diffractive cross section

\begin{tabular}{l|cccccr}
\hline \hline & \multicolumn{7}{|c}{ GLAUBER } \\
\hline & $\sigma_{\text {in }}^{N N}[\mathrm{mb}]$ & $\sigma_{\text {tot }}^{N A}[\mathrm{mb}]$ & $\sigma_{e l}^{N A}[\mathrm{mb}]$ & $\sigma_{\text {qel }}^{N A}[\mathrm{mb}]$ & $\sigma_{\text {in }}^{N A}[\mathrm{mb}]$ & $N_{\text {coll }}$ \\
\hline RHIC & 42.10 & 3297.56 & 1368.36 & 66.06 & 1863.14 & 4.70 \\
\hline LHC & 68.30 & 3850.63 & 1664.76 & 120.92 & 2064.95 & 6.88 \\
\hline \hline & \multicolumn{7}{c}{ GLAUBER+SRC } & & & \\
\hline & $\sigma_{\text {in }}^{N N}[\mathrm{mb}]$ & $\sigma_{\text {tot }}^{N A}[\mathrm{mb}]$ & $\sigma_{e l}^{N A}[\mathrm{mb}]$ & $\sigma_{q e l}^{N A}[\mathrm{mb}]$ & $\sigma_{\text {in }}^{N A}[\mathrm{mb}]$ & $N_{\text {coll }}$ \\
\hline RHIC & 42.10 & 3337.57 & 1398.08 & 58.47 & 1881.02 & 4.65 \\
\hline LHC & 68.30 & 3885.77 & 1690.48 & 112.65 & 2082.64 & 6.82 \\
\hline \hline & & & GLAUBER+SRC+GRIBOV $(q-2 q)$ & & & \\
\hline & $\sigma_{i n}^{N N}[\mathrm{mb}]$ & $\sigma_{\text {tot }}^{N A}[\mathrm{mb}]$ & $\sigma_{e l}^{N A}[\mathrm{mb}]$ & $\sigma_{q e l}^{N A}[\mathrm{mb}]$ & $\sigma_{\text {in }}^{N A}[\mathrm{mb}]$ & $N_{\text {coll }}$ \\
\hline RHIC & 42.10 & 3228.11 & 1314.04 & 71.99 & 1842.08 & 4.75 \\
\hline LHC & 68.30 & 3833.26 & 1655.70 & 113.37 & 2064.19 & 6.88
\end{tabular}

Table 2: Number of inelastic collisions $N_{\text {coll }}$ in $p-{ }^{208} \mathrm{~Pb}$ scattering at RHIC and LHC energies (after Ref. [10]).

$\sigma_{i n}^{N A}$ which, as shown in Table 2, is decreased by SRC and increased by Gribov IS. The effects of both SRC and Gribov IS amount to few percent in agreement with the results of the calculation of deuteron-gold scattering [8]. A further step in this type of calculations is to consider the high energy collision of two nuclei, $A$ and $B$; in this case the correlation contribution to the thickness function can be written as follows

$$
\begin{aligned}
& \Delta T_{A B}^{h}(b)=\frac{1}{\sigma_{t o t}^{N N}} A_{A} A_{B}^{2} \times \\
& \times \int d^{2} s_{A} \rho_{A}\left(\mathbf{s}_{A}\right) \int d^{2} s_{B 1} d^{2} s_{B 2} \Delta_{B}^{\perp}\left(\mathbf{s}_{B 1}, \mathbf{s}_{B 2}\right) \Gamma^{N N}\left(\mathbf{b}-\mathbf{s}_{A}+\mathbf{s}_{B 1}\right) \Gamma^{N N}\left(\mathbf{b}-\mathbf{s}_{A}+\mathbf{s}_{B 2}\right)+ \\
& +\{A \longleftrightarrow B\}
\end{aligned}
$$




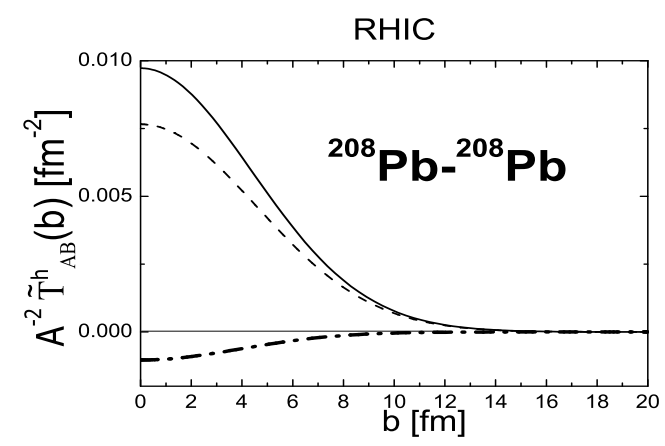

Figure 4: Uncorrelated thickness function $T_{A B}^{h}(b) / A^{2}$ (dash); correlation contribution $\Delta T_{A B}^{h}(b) / A^{2}$ (dotdash); total thickness function $\widetilde{T}_{A B}^{h} / A^{2}=\left[T_{A B}^{h}(b)-2 \Delta T_{A B}^{h}(b)\right] / A^{2}$ (full) in ${ }^{208} P b-{ }^{208} P b$ collisions at RHIC energies

where the 1st term describes the interaction of a nucleon in $A$ with two correlated nucleons in $B$ and the 2nd term in figure brackets viceversa. The thickness function including the effects of SRC in ${ }^{208} \mathrm{~Pb}-{ }^{208} \mathrm{~Pb}$ scattering at RHIC energies is shown in Fig. 3. Calculations of possible effects of SRC in heavy ion scattering are in progress.

\section{Acknowledgments}

I am grateful to Boris Kopeliovich, Irina Potashnikova, Ivan Schmidt, UTFSM, Massimiliano Alvioli and Chiara Benedetta Mezzetti, INFN, for a stimulating collaboration.

\section{References}

[1] R. J. Glauber, in Lectures in Theoretical Physics, W. E. Brittin et al Editors, New York (1959).

[2] S. C. Pieper, R. B. Wiringa, V. R. Pandharipande, Phys. Rev.C46 (1992) 1741.

[3] R. Subedi, R. Shneor, P. Monaghan, B. D. Anderson, et al., Probing Cold Dense Nuclear Matter, Science 320, 1476 (2008) [nucl-ex 0908.1514].

[4] V. N. Gribov, Sov. JETP 29 (1969) 483.

[5] M. Alvioli, C. Ciofi degli Atti, I. Marchino, V. Palli, H. Morita Phys. Rev.C78 (2008) 031601(R).

[6] M. Alvioli, C. Ciofi degli Atti, B. Z. Kopeliovich, I. K. Potashnikova, I. Schmidt, Phys. Rev. C81 (2010) 025204.

[7] B. Z. Kopeliovich, L. I. Lapidus, A. B. Zamolodchikov, JETP Lett. 33 (1981) 595. [Pisma Zh. Eksp. Teor. Fiz. 33 (1981) 595].

[8] B. Z. Kopeliovich, Phys. Rev. C68 (2003) 044906.

[9] B. Z. Kopeliovich, I. K. Potashnikova and I. Schmidt Phys. Rev. C73 (2006) 034901.

[10] C. Ciofi degli Atti, C. B. Mezzetti, B. Z. Kopeliovich, I. K. Potashnikova, I. Schmidt, Phys. Rev. C84 (2011) 025205 [nucl-th 1105.1080v2].

[11] M. Alvioli, C. Ciofi degli Atti, H. Morita, Phys. Rev. Lett. 100 (2008) 162503. 